\title{
HLA-associated susceptibility to Type 2 (non-insulin-dependent) diabetes mellitus: the Wadena City Health Study
}

\author{
S.S. Rich ${ }^{1}$, L. R.French ${ }^{2}$, J.M.Sprafka ${ }^{3}$, J.P.Clements ${ }^{2}$ and F.C. Goetz ${ }^{4}$ \\ ${ }^{1}$ Department of Laboratory Medicine and Pathology and Institute of Human Genetics; \\ ${ }^{2}$ Chronic Disease and Environmental Epidemiology Section, Minnesota Department of Health; \\ ${ }^{3}$ Division of Epidemiology, and ${ }^{4}$ Department of Medicine, University of Minnesota, Minneapolis, Minnesota, USA
}

\begin{abstract}
Summary. Epidemiologic data suggest that a parental history of Type 2 (non-insulin-dependent) diabetes mellitus increases the risk of Type 1 (insulin-dependent) diabetes in siblings of a Type 1 diabetes proband. This increase in risk is consistent with a shared genetic susceptibility between Type 1 and Type 2 diabetes. We have previously reported evidence that HLA-DR4-linked factors may represent a homogeneous subset of diabetes susceptibility. First, HLADR4 frequency was higher in Type 1 diabetic study subjects with a Type 2 diabetic parent than in Type 1 diabetic subjects whose parents were not diabetic. Second, a DR4-haplotype was transmitted from the Type 2 diabetic parent to the Type 1 offspring more often than expected. These data are consis-
\end{abstract}

tent with the hypothesis that families with a Type 2 diabetic parent and Type 1 diabetic child, heavily determined by HLA-DR4 linked factors, may represent a homogeneous subset of diabetes susceptibility. In this report, we further explore the relationship between the high-risk HLA antigen (HLA-DR4) in study subjects with differing glycaemic status (National Diabetes Data Group criteria). In this communitybased study, we find evidence that HLA-DR4 is increased in study subjects with Type 2 diabetes and may be a marker for Type 2 diabetes susceptibility.

Key words: Genetics, Type 2 (non-insulin-dependent) diabetes mellitus, HLA.
The cause of Type 2 (non-insulin-dependent) diabetes is likely to be multifactorial in nature, consisting of both genetic and familial factors contributing to susceptibility and modified by exogenous environmental risk factors. The most powerful predictor of risk for Type 2 diabetes is being an identical (monozygotic, or MZ) twin of a diabetic subject. The MZ twin concordance for Type 2 diabetes has a life-time risk that approaches $100 \%$ [1], indicating that the susceptibility is highly familial, with disease determined by genetic or common environmental factors or a combination of the two. Although the MZ twin concordance may suggest that a few major gene effects could contribute to the susceptibility to Type 2 diabetes, no single genetic contributor has been identified to date which can account for more than a few percent of cases.

Although many investigations operationally consider Type 1 (insulin-dependent) diabetes as distinct from Type 2 diabetes, it does not necessarily follow that the risk factors (genetic or environmental) for Type 1 diabetes are distinct from those for Type 2 diabetes. Epidemiologic data suggest that having a parent with Type 2 diabetes increases the risk for Type 1 diabetes in siblings of a Type 1 diabetic proband $[2,3]$. This increase in risk is consistent with a shared genetic susceptibility between Type 1 and
Type 2 diabetes. We previously evaluated genetic risk factors in families having Type 1 diabetic study subjects only and compared them with families having at least one Type 1 diabetic child and at least one Type 2 diabetic parent. We previously found evidence [4] that the frequency of a high risk HLA antigen (HLA-DR3) was lower in Type 1 diabetic study subjects with a Type 2 diabetic parent than in Type 1 diabetic study subjects with nondiabetic parents. The increased frequency of HLA-DR4 was maintained in these families with an increased rate of transmission of the HLA-DR4 allele from Type 2 diabetic parent to Type 1 diabetic offspring. These results [4] indicated that families with a Type 2 diabetic parent and Type 1 diabetic child, heavily determined by HLA-DR4 linked factors, either represent a subset of diabetes susceptibility or represent a part of a continuum of the diabetic spectrum.

The Wadena City Health Study was established to provide further insight into the relationship between pancreatic function, Type 2 diabetes, impaired glucose tolerance (IGT), and aging [5] in a population of Northern European ancestry. The members of the population participating in the study include all known diabetic subjects and a stratified (by age and gender) random sample of all other 
Table 1. Distribution of all Wadena City Health Study subjects by gender and current glycaemic status

\begin{tabular}{lllll}
\hline Gender & NGT & IGT & $\begin{array}{l}\text { Type 2 } \\
\text { diabetes }\end{array}$ & $\begin{array}{l}\text { Type 1 } \\
\text { diabetes }\end{array}$ \\
\hline Male & 159 & 17 & 38 & 3 \\
Female & 185 & 12 & 59 & 3 \\
Total & 344 & 29 & 97 & 6 \\
\hline
\end{tabular}

NGT, Normal glucose tolerance; IGT, impaired glucose tolerance

residents over the age of 20 years, based upon a city wide census. The prevalence of Type 2 diabetes $(7.8 \%)$ and IGT $(5.2 \%)$ in this community are consistent with estimates from the National Health and Nutrition Examination Survey II ( $6.6 \%$ and $4.6 \%$, respectively) using the National Diabetes Data Group (NDDG) criteria [6]. Although there is evidence that a substantial proportion of the population has some evidence of hyperglycaemia, the community represents an excellent resource for evaluating specific hypotheses concerning both genetic and environmental factors contributing to diabetes and its complications.

In order to investigate whether there is an association between the HLA system and Type 2 diabetes, we have examined HLA antigens in Type 2 diabetic subjects and compared them with the HLA antigen frequencies in a stratified random sample of normoglycaemic individuals. This paper provides further evidence that genes in the HLA region may have some influence in genetic susceptibility to Type 2 diabetes.

\section{Subjects and methods}

\section{Subjects}

Study subjects were selected from a sampling frame including all physician-diagnosed diabetic residents of Wadena, Minnesota (population 4699, 1980 census), and all other residents who were 20 years of age or older. The population of Wadena, Minnesota, is almost exclusively derived from Northern European ancestry. In our sample, $99.2 \%$ are Caucasian, $0.4 \%$ Native American, and $0.4 \%$ Oriental. Of the Caucasian subjects, the major ethnic backgrounds are reported to be German (47\%), Norwegian (15\%), English (9\%), Swedish (8\%), Irish (4\%) and Finnish (4\%).

All physician-diagnosed diabetic subjects were invited to participate in the study, and a stratified random sample of all other residents was asked to participate. Standard study procedures that had been approved by the University of Minnesota Committee on the Use of Human Subjects in Research were followed in recruiting all study subjects.

The original study design differentiated the study subjects according to the source of sampling; i. e. each subject was a member identified as either a previously diagnosed diabetic or a member of a stratified random sample [5]. For this study, the subjects were grouped into four distinct groups: "normal" glucose tolerance, impaired glucose tolerance (IGT), Type 2 diabetes, and Type 1 diabetes. The distribution of the study subjects by gender and four groups of glycaemic status is shown in Table 1 . The 344 subjects with normal glucose tolerance include 334 subjects from the stratified random sample and 10 subjects (mean age $78.9 \pm 3.4$ years) who were originally diagnosed as diabetic but had normal glucose tolerance by current testing and NDDG criteria. The mean age at sampling for those subjects with normal glucose tolerance was $47.1 \pm 1.1$ years. Within the group of 29 study subjects with IGT,
24 were identified from the stratified random sample and 5 had previously been diagnosed as diabetic. The 97 Type 2 diabetic subjects include 24 identified from the stratified random sample, 7 who were treated outside of Wadena, and 66 from the previously known group. The average age of sampling for those Type 2 diabetic subjects was $66.0 \pm 1.4$ years. Six previously identified diabetic study subjects were found to have Type 1 diabetes (the prevalence of Type 1 diabetes in this community is $0.3 \%$ ). The subjects with Type 1 diabetes (mean age at diagnosis of $25.2 \pm 5.7$ years) were excluded from further analyses. HLA typing was available on five of the six Type 1 diabetic subjects; two were HLA-DR4-negative $(2 / 3,3 / 10)$ and three were HLA-DR4-positive $(1 / 4,3 / 4,4 / 4)$.

\section{Data collection}

Subjects participated in two mornings of testing and data collection within a 10-day period. Diet preparation occurred before each testing day. Subjects continued taking medications not related to diabetes and those subjects taking insulin or oral hypoglycaemic agents were requested to delay these medications until after the day's tests were completed. Two metabolic tests of carbohydrate tolerance, a 75-g oral glucose tolerance test (OGTT) and a standard liquid meal, were randomly assigned to two test mornings. The standard liquid meal, designated as the Ensure-Plus challenge test (EPCT), consisted of $480 \mathrm{ml}$ of Ensure-Plus (Ross Laboratories, Columbus, Ohio, USA) containing $710 \mathrm{kcal}$ ( $53 \%$ carbohydrate, $15 \%$ protein, and $32 \%$ fat).

The OGTT followed the procedure recommended by the NDDG with blood drawn $5 \mathrm{~min}$ and $1 \mathrm{~min}$ before the 75 -g glucose challenge, and at 30, 60,90, and $120 \mathrm{~min}$ after ingestion [7]. Fasting and 90-min post-meal blood samples for glucose and C-peptide were taken for the EPCT.

Typing for HLA-DR antigens was performed in an unbiased, blinded protocol without regard to glycaemic state. HLA-DR antigens were determined on all available subjects. Based upon initial ascertainment of study subjects, HLA typing was performed on $90.8 \%$ of subjects with normal glucose tolerance and $88.7 \%$ of subjects with Type 2 diabetes. All HLA typing was performed in the HLA laboratory of the University of Minnesota Hospitals and Clinics by published methods [8].

\section{Diagnostic criteria}

NDDG criteria, modified for an epidemiologic setting, were used to assess the current diabetic status for both previously diagnosed diabetic patients and subjects from the stratified random sample [7, 9]. Subjects with fasting glucose concentrations in venous plasma greater than $7.8 \mathrm{mmol} / 1(140 \mathrm{mg} / \mathrm{dl})$ or a sustained glucose elevation greater than $11.1 \mathrm{mmol} / \mathrm{l}(200 \mathrm{mg} / \mathrm{dl})$ were considered diabetic by NDDG standards. A glucose concentration greater than $11.1 \mathrm{mmol} / \mathrm{l}$ at both 2 -h after challenge, and at 30,60 , or $90 \mathrm{~min}$ after glucose challenge was considered sustained elevation. Those with a 2-h post-challenge glucose concentration between 7.8 and $11.1 \mathrm{mmol} / \mathrm{l}$, but with a glucose concentration greater than $11.1 \mathrm{mmol} / \mathrm{l}$ at 30,60 , or $90 \mathrm{~min}$ post-challenge were considered to have IGT by NDDG standards.

Previously diagnosed diabetic subjects were classified as Type 1 diabetic if they had been identified with definite or probable Type 1 diabetes in medical records and had a plasma C-peptide level less than $0.2 \mathrm{nmol} / 190 \mathrm{~min}$ after the mixed meal challenge test (EPCT). This C-peptide concentration is consistent with the definition of Type 1 diabetes for the Diabetes Control and Clinical Trial [10].

\section{Statistical analysis}

Prevalence ratios and associated chi-square tests were used to assess the association between HLA-DR4 and the glycaemic status of different groups of subjects from the Wadena City Health Study [11]. 
Table 2. HLA-DR4 antigen status in Wadena City Health Study subjects with Type 2 diabetes or with normal glucose tolerance (NGT)

\begin{tabular}{lll}
\hline HLA-DR4 & \multicolumn{2}{l}{ Glycaemic status } \\
\cline { 2 - 3 } & NGT & Type 2 diabetes \\
\hline Absent & $166(73 \%)$ & $50(58 \%)$ \\
Present & $62(27 \%)$ & $36(42 \%)$ \\
\hline
\end{tabular}

Prevalence ratio $=1.93 . \chi^{2}=5.59$, with continuity correction $(p=0.02)$

Table 3. Frequencies of HLA-DR antigens in Wadena City Health Study subjects with normal glucose tolerance (NGT) or Type 2 diabetes

\begin{tabular}{lll}
\hline HLA antigen & \multicolumn{2}{l}{ Glycaemic status } \\
\cline { 2 - 3 } & NGT & Type 2 diabetes \\
\hline DR1 & $53(23 \%)$ & $22(26 \%)$ \\
DR2 & $63(28 \%)$ & $28(33 \%)$ \\
DR3 & $53(23 \%)$ & $13(15 \%)$ \\
DR4 & $62(27 \%)$ & $36(42 \%)^{a}$ \\
DR5 & $34(15 \%)$ & $10(12 \%)$ \\
DR6 & $59(26 \%)$ & $19(22 \%)$ \\
DR7 & $53(23 \%)$ & $16(19 \%)$ \\
all others & $40(18 \%)$ & $13(15 \%)$ \\
\hline
\end{tabular}

${ }^{\mathrm{a}} p<0.05$

The statistical software used for these analyses was SPSS [12]. Since previous results suggested that HLA-DR4 was implicated in diabetic susceptibility, all analyses were performed on the basis of presence or absence of this antigen; thus, no corrections for analyses using multiple antigens were required.

\section{Results}

A total of 425 of 476 subjects were typed for HLA-DR antigens. HLA-DR typing was available for 228 of 251 subjects with normal glucose tolerance from our stratified random sample and on 86 of 97 Type 2 diabetic patients. Thus, 314 of the 476 subjects with either normal glucose tolerance $(n=228)$ or with Type 2 diabetes $(n=$ 86) were typed for HLA-DR antigens and used in these analyses. We compared the presence or absence of HLADR4 in subjects with normal glucose tolerance with Type 2 diabetic subjects (Table 2). Of the 86 subjects with Type 2 diabetes, $36(42 \%)$ were HLA-DR4 positive. Of the 228 subjects with normal glucose tolerance, $62(27 \%)$ were positive for the HLA-DR4 antigen. The prevalence of the HLA-DR4 antigen was significantly higher in Type 2 diabetic patients relative to subjects with normal glucose tolerance (prevalence ratio of 1.9 , chi-square = 6.59 with continuity correction, $p=0.02$ ). In light of this finding, we then determined the presence or absence of other common HLA-DR specificities in this population. There was no other antigen tested that was significantly more or less frequent in the Type 2 diabetic patients than in the subjects with normal glucose tolerance, although HLA-DR3 appeared to be at reduced frequency in the Type 2 diabetic patients (Table 3 ). These data provide evidence that genes in the HLA region may influence in genetic susceptibility to Type 2 diabetes.
Profiles of each group of subjects by C-peptide, body mass index (BMI), fasting plasma glucose, and family history of diabetes were evaluated with respect to HLA-DR4 status (Table 4). Within the group of 228 subjects with normal glucose tolerance, there was no difference in C-peptide $(2.9 \pm 0.1 \mathrm{nmol} / \mathrm{l}$ vs $2.9 \pm 0.2 \mathrm{nmol} / \mathrm{l})$, BMI $\left(26.5 \pm 0.4 \mathrm{~kg} / \mathrm{m}^{2}\right.$ vs $\left.26.2 \pm 0.6 \mathrm{~kg} / \mathrm{m}^{2}\right)$, fasting plasma glucose $(5.2 \pm 0.0 \mathrm{mmol} / 1$ vs $5.3 \pm 0.1 \mathrm{mmol} / \mathrm{l})$, or percentage of subjects reporting a family history of diabetes $(30.3 \%$ vs $22.2 \%$ ) with respect to absence or presence of HLADR4, respectively. Within the Type 2 diabetic patients, there was no difference in C-peptide $(3.6 \pm 0.3 \mathrm{nmol} / \mathrm{l}$ vs $3.4 \pm 0.4 \mathrm{nmol} / 1)$, BMI $\left(30.8 \pm 1.0 \mathrm{~kg} / \mathrm{m}^{2}\right.$ vs $29.7 \pm 1.0 \mathrm{~kg} /$ $\left.\mathrm{m}^{2}\right)$, fasting plasma glucose $(8.8 \pm 0.5 \mathrm{mmol} / \mathrm{l}$ vs $9.4 \pm$ $0.5 \mathrm{mmol} / \mathrm{l})$ or family history $(60.5 \%$ vs $59.4 \%)$ with respect to absence or presence of HLA-DR4, respectively.

The Type 2 diabetic subjects had, on average, higher mean C-peptide levels, greater BMI, and higher fasting plasma glucose levels than the subjects with normal glucose tolerance, as expected. The group of Type 2 diabetic subjects reported a markedly increased frequency of either a parent or sibling with diabetes $(60 \%)$ in contrast to the observed in the normal glucose tolerance subjects $(30.3 \%)$. Although HLA-DR4 is increased in Type 2 diabetic subjects vs subjects with normal glucose tolerance, the clinical correlates presented here are not significantly influenced by the presence or absence of the HLADR4 allele.

\section{Discussion}

Type 1 diabetes is thought to be aetiologically distinct from Type 2 diabetes. Type 1 diabetes is considered to be autoimmune in origin and is clearly associated with specific HLA antigens and demonstrates linkage of a major susceptibility locus with the HLA complex [13]. Type 2 diabetes is thought not to be autoimmune and has not shown a consistent association or linkage with genes in the

Table 4. HLA-DR4 antigen status in Wadena City Health Study subjects by mean plasma C-peptide, body mass index (BMI), fasting plasma glucose (FPG) and reported family history of diabetes

\begin{tabular}{llcc}
\hline & HLA-DR4 & \multicolumn{2}{c}{ Glycaemic status } \\
\cline { 2 - 4 } & & Normal & Type 2 diabetes \\
\hline $\begin{array}{l}\text { C-peptide } \\
\begin{array}{l}90 \text {-min } \\
\text { post-meal } \\
\text { (nmol/1) }\end{array}\end{array}$ & Absent & $2.9(0.1)$ & $3.6(0.3)$ \\
$\begin{array}{l}\text { BMI } \\
\left(\mathrm{kg} / \mathrm{m}^{2}\right)\end{array}$ & Absent & $26.5(0.4)$ & $30.8(1.0)$ \\
FPG & Present & $26.2(0.6)$ & $29.7(1.0)$ \\
& Absent & $5.2(0.0)$ & $8.8(0.5)$ \\
& Present & $5.3(0.1)$ & $9.4(0.5)$ \\
$\begin{array}{l}\text { Family } \\
\text { history }\end{array}$ & Absent & $30.3 \%$ & $60.5 \%$ \\
& Present & $22.2 \%$ & $59.1 \%$ \\
\hline
\end{tabular}

FPG, mean of two values taken on the day of oral glucose tolerance testing $(\mathrm{mmol} / \mathrm{l})$. Mean $( \pm$ SEM $)$ 
HLA region. On the other hand, there is an increased risk for Type 1 diabetes in siblings when a Type 1 diabetic proband has a Type 2 diabetic parent $[2,3]$. Taken together, there is evidence of a genetic relationship between Type 1 and Type 2 diabetes that is mediated, in part, through genes in the HLA region.

Separate major genes have been hypothesized as being responsible for Type 1 and Type 2 diabetes susceptibility. These major locus models are attractive for several reasons. The primary reason is that a major locus has a high probability of being mapped to a chromosomal location. Although genetic susceptibility to Type 1 diabetes is strongly influenced by genes in the HLA region of human chromosome 6 , not all of the susceptibility is explained by this one region. When two siblings have Type 1 diabetes, $55 \%$ will have the same HLA region genes (haplotypes), $40 \%$ of the sibling pairs will have only one haplotype in common, while $5 \%$ of the sibling pairs will have no HLA haplotypes in common [14]. Although a multifactorial source of genetic liability to either Type 1 or Type 2 diabetes or both is a concern for gene mapping, there is no need to imply that multifactorial inheritance implies hundreds of loci. Rather, there remains the more likely outcome that Type 1 and Type 2 diabetes are each the result of the actions of a limited number of genes (a major gene and some genes with small effect). Thus, some overlap may occur in the loci required for at least some forms of Type 1 diabetes and Type 2 diabetes [15].

A recent study [16] has demonstrated an increased serum viscosity in Type 2 diabetic patients with microvascular disease that has been linked to changes in serum protein composition. The major change observed is a decline in albumin and in increase in the levels of acute-phase proteins (including haptoglobin). Acute-phase proteins were also increased in advanced glucose intolerance as well as in established Type 2 diabetes, although there was no relationship with degree of control or duration of diabetes. Although this effect may be due to metabolic changes (increased blood glucose), the examination of families with both Type 2 and Type 1 diabetes with respect to genetic markers could be highly informative in defining mutual risk factors.

In a series of studies $[17,18]$, islet cell antibodies (ICA) and thyrogastric antibodies were determined in 204 patients with Type 2 diabetes treated with diet or oral hypoglycaemic agents or both (non-insulin-requiring, NIR) and in 108 age-matched patients who were being treated with insulin (insulin-requiring, IR). HLA-DR antigens were determined in only $127 \mathrm{NIR}$ and 69 IR patients. The IR patients differed from the NIR patients with respect to higher frequency of ICA, thyroid antibodies, and HLADR4 frequency. Patients who were heterozygous for HLA-DR3/DR4 showed significantly higher frequency of ICA and complement-fixing ICA than patients without the heterozygous form DR3/DR4, although no corrections for multiple antigens tested were made. These data suggest that Type 2 diabetes is a heterogeneous disorder including at least two major subgroups, characterized by HLA-DR antigens and organ-specific antibodies.

The role of genetic markers other than HLA in contributing to susceptibility to both Type 1 diabetes and Type 2 diabetes has been equivocal. In a Mexican American population randomly selected from three San Antonio neighbourhoods, statistically significant associations were found between Type 2 diabetes and the $\mathrm{Rh}$ blood type and haptoglobin phenotype $[19,20]$. The haptoglobin association showed a dose effect with a single dose of the haptoglobin- 1 allele associated with a $50 \%$ increase in Type 2 diabetes prevalence and a haptoglobin 1-1 homozygote was associated with a $100 \%$ increase in Type 2 diabetes prevalence. In a separate study of Type 2 diabetes and seven polymorphic markers using 398 Caucasian subjects (97 Type 2 diabetic subjects and 301 subjects with normal glucose tolerance) and 390 Hispanic subjects (191 Type 2 diabetic subjects and 199 subjects with normal glucose tolerance), there was no association with either haptoglobin or $\mathrm{Rh}$ [21]. In families with at least one Type 2 diabetic parent and a Type 1 diabetic child, the haptoglobin genotype does not appear to be a significant contributor to diabetes susceptibility, either with respect to association with diabetes or with respect to linkage with a secondary susceptibility locus [4].

In summary, we present data that are consistent with an HLA-DR4 effect in the susceptibility to Type 2 diabetes, thereby providing a common shared factor responsible for genetic susceptibility at least in a portion of families. The supporting data include the previously reported significantly cumulative risk for Type 1 diabetes in siblings of Type 1 diabetic probands with a Type 2 diabetic parent and the increased relative risk in these families for Type 1 diabetes associated with HLA-DR4 but not HLA-DR3. These results provide evidence that HLA-DR4 linked factors may represent a major component of general diabetes susceptibility. The evidence is particularly cogent, since it comes from a population-based sample, rather than selected patients seen at a referral (medical) centre. These data, therefore, support the possibility of better assessing the relative contribution of hereditary and environmental determinants in the aetiology of Type 2 diabetes.

Acknowledgements. We thank Ms H. Noreen for the HLA serologic typing. This study was supported in part by grants from the American Diabetes Association and by N.I.H. grants DK33225 and RR00400.

\section{References}

1. Newman B, Selby JV, King MC, Slemenda C, Fabsitz R, Friedman GD (1987) Concordance for type 2 (non-insulin-dependent) diabetes mellitus in male twins. Diabetologia 30: 763-768

2. Chern MM, Anderson VE, Barbosa J (1982) Empirical risk for insulin-dependent diabetes (IDD) in sibs. Further definition of genetic heterogeneity. Diabetes 31: 1115-1118

3. Wagener DK, Sacks JM, LaPorte RE, Macgregor JM (1982) The Pittsburgh study of insulin-dependent diabetes mellitus. Risk for diabetes among relatives of IDDM. Diabetes 31: 136-144

4. Rich SS, Panter SS, Goetz FC, Hedlund B, Barbosa J (1991) Shared genetic susceptibility of type 1 (insulin-dependent) and type 2 (non-insulin-dependent) diabetes mellitus: contributions of HLA and haptoglobin. Diabetologia 34: 350-355

5. French LR, Boen JR, Martinez AM, Bushhouse SA, Sprafka JM, Goetz FC (1990) A population-based study of impaired glucose tolerance and type II diabetes: the Wadena City Health Study. Diabetes 39: 1131-1137 
6. Harris MI, Hadden WC, Knowler WC, Bennett PH (1987) Prevalence of diabetes and impaired glucose tolerance and plasma glucose levels in U.S. population aged 20-74 yr. Diabetes 36: 523-534

7. National Diabetes Data Group (1979) Classification and diagnosis of diabetes mellitus and other categories of glucose intolerance. Diabetes 28: 1039-1057

8. Barbosa J, Bach FH, Rich SS (1982) Genetic heterogeneity of diabetes and HLA. Clin Genet 21:25-32

9. Harris MI, Entmacher P (1985) Classification and diagnostic criteria for diabetes and other categories of glucose intolerance. In: Harris M, Hamman RF (eds) Diabetes in America. U.S. Government Printing Office. Washington, D.C., Chap. 2

10. DCCT Research Group (1987) Effects of age, duration and treatment of insulin-dependent diabetes mellitus on residual beta cell function: observations during eligibility testing for the Diabetes Control and Complications Trial (DCCT). J Clin Endocrinol Metab 65: 30-36

11. Fleiss JL (1981) Statistical methods for rates and proportions. Wiley, New York

12. SPSS (1990) SPSS-X Users guide. 3rd edn. SPSS, Chicago

13. Nerup J, Platz P, Andersen OO et al. (1974) HL-A antigens and diabetes mellitus. Lancet II: 864-866

14. Spielman RS, Baker L, Zmijewski CM (1980) Gene dosage and susceptibility to insulin-dependent diabetes. Ann Hum Gent 44: $135-150$

15. Rich SS (1990) Mapping genes in diabetes: a genetic epidemiological perspective. Diabetes 39: 1315-1319

16. McMillan DE (1989) Increased levels of acute-phase serum proteins in diabetes. Metabolism 38: 1042-1046
17. Groop L, Koskimies S, Pelkonen R, Tolppanen EM (1983) Increased frequency of HLA-Cw4 in type 2 diabetes. Acta Endocrinol (Copenh) 104: 475-478

18. Groop L, Miettinen A, Groop PH, Meri S, Koskimies S, Bottazzo GF (1988) Organ-specific autoimmunity and HLA-DR antigens as markers for beta-cell destruction in patients with type II diabetes. Diabetes 37: 99-103

19. Chakraborty R, Ferrell RE, Stern MP, Haffner SM, Hazuda HP, Rosenthal M (1986) Relationship of prevalence of non-insulindependent diabetes mellitus to Amerindian admixture in the Mexican Americans of San Antonio, Texas. Genet Epidemiol 3: 435-454

20. Stern MP, Ferrell RE, Rosenthal M, Haffner SM, Hazuda HP (1986) Association between NIDDM, Rh blood group, and haptoglobin phenotype. Results from the San Antonio Heart Study. Diabetes 35: 387-391

21. Iyengar S, Hamman RF, Marshall JA, Baxter J, Majumder PP, Ferrell RE (1989) Genetic studies of type 2 (non-insulin-dependent) diabetes mellitus: lack of association with seven genetic markers. Diabetologia 32: 690-693

Received: 9 June 1992

and in revised form: 19 October 1992

Dr. S.S. Rich

Box 206 U.M.H.C.

Institute of Human Genetics

University of Minnesota

Minneapolis, MN 55455

USA 